\title{
Mass and Isospin Effects in Multifragmentation
}

C. Sfienti ${ }^{\mathrm{ab}}$, P. Adrich ${ }^{\mathrm{a}}$, T. Aumann ${ }^{\mathrm{a}}$, C.O. Bacri ${ }^{\mathrm{c}}$, T. Barczyk ${ }^{\mathrm{d}}$, R. Bassini ${ }^{\mathrm{e}}$, C. Boiano $^{\mathrm{e}}$, A.S. Botvina ${ }^{\text {af }}$, A. Boudard ${ }^{g}$, J. Brzychczyk $^{\mathrm{d}}$, A. Chbihi ${ }^{\mathrm{h}}$, J. Cibor ${ }^{\mathrm{i}}$, B. Czech ${ }^{\mathrm{i}}$, M. De Napoli ${ }^{\text {, }}$ J.-E. Ducret ${ }^{\mathrm{g}}$, H. Emling ${ }^{\mathrm{a}}$, J. Frankland ${ }^{\mathrm{h}}$, M. Hellström ${ }^{\mathrm{a}}$, D. Henzlova ${ }^{\mathrm{a}}$, K. Kezzara ${ }^{\mathrm{a}}$, G. Imméb ${ }^{\mathrm{b}}$ I. Iorie ${ }^{\mathrm{e}}$ H. Johansson ${ }^{\mathrm{a}}$, A. Lafriakh ${ }^{\mathrm{c}}$, A. Le Fèvre ${ }^{\mathrm{a}}$, E. Le Gentil ${ }^{\mathrm{g}}$, Y. Leifels ${ }^{\mathrm{a}}$, W.G. Lynch ${ }^{\mathrm{j}}$, J. Lühning ${ }^{\mathrm{a}}$, J. Lukasik ${ }^{\mathrm{a} i}$, U. Lynen ${ }^{\mathrm{a}}$, Z. Majka ${ }^{\mathrm{d}}$, M. Mocko ${ }^{\mathrm{j}}$, W.F.J. Müller ${ }^{\mathrm{a}}$, A. Mykulyak ${ }^{\mathrm{k}}$, H. Orth ${ }^{\mathrm{a}}$, A.N. Otte ${ }^{\mathrm{a}}$, R. Palit ${ }^{\mathrm{a}}$, A. Pullia ${ }^{\mathrm{e}}$, G. Raciti ${ }^{\mathrm{b}}$, E. Rapisarda ${ }^{\mathrm{b}}$, H. Sann ${ }^{\mathrm{a}}$, C. Schwarz ${ }^{\mathrm{a}}$, H. Simon ${ }^{\mathrm{a}}$, A. Sokolov ${ }^{\mathrm{a}}$, K. Sümmerer ${ }^{\mathrm{a}}$, W. Trautmann ${ }^{\mathrm{a}}$, M.B. Tsang ${ }^{\mathrm{j}}$, G. Verde ${ }^{\mathrm{j}}$, C. Volant ${ }^{\mathrm{g}}$, M. Wallace ${ }^{\mathrm{j}}$, H. Weick ${ }^{\mathrm{a}}$, J. Wiechula ${ }^{\mathrm{a}}$, A. Wieloch ${ }^{\mathrm{d}}$ and B. Zwieglinski ${ }^{\mathrm{k}}$

${ }^{a}$ Gesellschaft für Schwerionenforschung, D-64291 Darmstadt, Germany

bDipartimento di Fisica dell'Universitá and LNS-INFN, I-95126 Catania, Italy

'Institut de Physique Nucléaire, IN2P3-CNRS et Université, F-91406 Orsay, France

${ }^{\mathrm{d}}$ M. Smoluchowski Institute of Physics, Jagiellonian Univ., Pl-30059 Kraków, Poland

eIstituto di Scienze Fisiche, Università degli Studi and INFN, I-20133 Milano, Italy

${ }^{\mathrm{f}}$ Inst. Nucl. Res., Russian Accademy of Science, Ru-117312 Moscow, Russia

gDAPNIA/SPhN, CEA/Saclay, F-91191 Gif-sur-Yvette, France

${ }^{\text {h}}$ GANIL, CEA et IN2P3-CNRS, F-14076 Caen, France

${ }^{i}$ H. Niewodniczański Institute of Nuclear Physics, Pl-31342 Kraków,Poland

${ }^{j}$ Department of Physics and Astronomy and NSCL, MSU, East Lansing, MI 48824, USA

${ }^{k}$ A. Sołtan Institute for Nuclear Studies, Pl-00681 Warsaw, Poland

A systematic study of isospin effects in the breakup of projectile spectators at relativistic energies has been performed with the ALADiN spectrometer at the GSI laboratory (Darmstadt). Four different projectiles ${ }^{197} \mathrm{Au},{ }^{124} \mathrm{La},{ }^{124} \mathrm{Sn}$ and ${ }^{107} \mathrm{Sn}$, all with an incident energy of $600 \mathrm{AMeV}$, have been used, thus allowing a study of various combinations of masses and $N / Z$ ratios in the entrance channel.

The measurement of the momentum vector and of the charge of all projectile fragments with $Z>1$ entering the acceptance of the ALADiN magnet has been performed with the high efficiency and resolution achieved with the TP-MUSIC IV detector.

The Rise and Fall behavior of the mean multiplicity of IMFs as a function of $Z_{\text {bound }}$

*deceased 
and its dependence on the isotopic composition has been determined for the studied systems. Other observables investigated so far include mean $N / Z$ values of the emitted light fragments and neutron multiplicities. Qualitative agreement has been obtained between the observed gross properties and the predictions of the Statistical Multifragmentation Model.

\section{Introduction}

The role of isospin in multifragmentation has been explored very little up to now. This is in striking contrast to the importance of isospin, in particular for any interpretation of multifragmentation as a manifestation of the liquid-gas phase transition in nuclear matter. Müller and Serot, in their seminal paper [1], have demonstrated that the two-fluid nature of nuclear matter has very specific consequences for the phase behavior in the coexistence region. Different isotopic compositions are predicted for the coexisting liquid and gas phases, with the gas being more neutron rich than the liquid in asymmetric matter. This difference stems from the decrease in the symmetry energy in nuclear matter as the density is decreased. The expected magnitude of this density dependence, however, is model dependent and very poorly constrained by existing data [2].

Furthermore, the calculations are restricted to infinite matter with neither Coulomb forces nor fragment formation included. In addition, the assumed isotopic composition is typically varied within a range of proton fractions whose limits are not easily accessible in experiments with heavy nuclei. Theoretical studies for finite systems also indicate that the sequential decay of excited reaction products has a tendency to modify some of the expected effects [3]. Some observables are, however, predicted to be robust with respect to sequential decay. If chemical equilibrium can be assumed, isotopic ratios can be constructed whose exponential dependence on the ratio $\mu / T$ of the chemical potential $\mu$ and the temperature $T$ will amplify differences resulting from the variation of $\mu$ with the isotopic composition. Measured isotopic yield ratios were found to vary strongly with the $N / Z$ ratio of the emitting source [4], in agreement with this expectation $[5,6]$. Recently, significant differences between neutron-rich and neutron-poor systems have been observed [7]. In particular, the strong enhancement of the neutron content in the 'gas' with increasing $N / Z$ of the system has been interpreted as being in qualitative agreement with the predictions of [1]. The role of the excitation energy has also been emphasized [8] and evidence has been found for the increased production of neutron-rich isotopes with excitation energy that is predicted by the Statistical Multifragmentation Model (SMM, ref. [9]).

The SMM predictions for the fragmentation of two $A=124$ systems, one neutron-rich $(N / Z=1.48)$ and one neutron-poor $(N / Z=1.18)$, are given in Fig. 1. The isotopic compositions of the hot fragments produced at breakup are globally very different for the two systems, close to the $N / Z$ ratios of the primary projectiles which they approach with increasing mass number (top panels). The overall mass dependence is rather weak, with a dependence on excitation energy predicted to be different for the neutron-rich and neutron-poor cases. The calculated mass yield curves are fairly similar for these systems except at low excitation energy (bottom panels of Fig. 1). Exploring these dependences experimentally will be important for our understanding of the role of phase space in the 

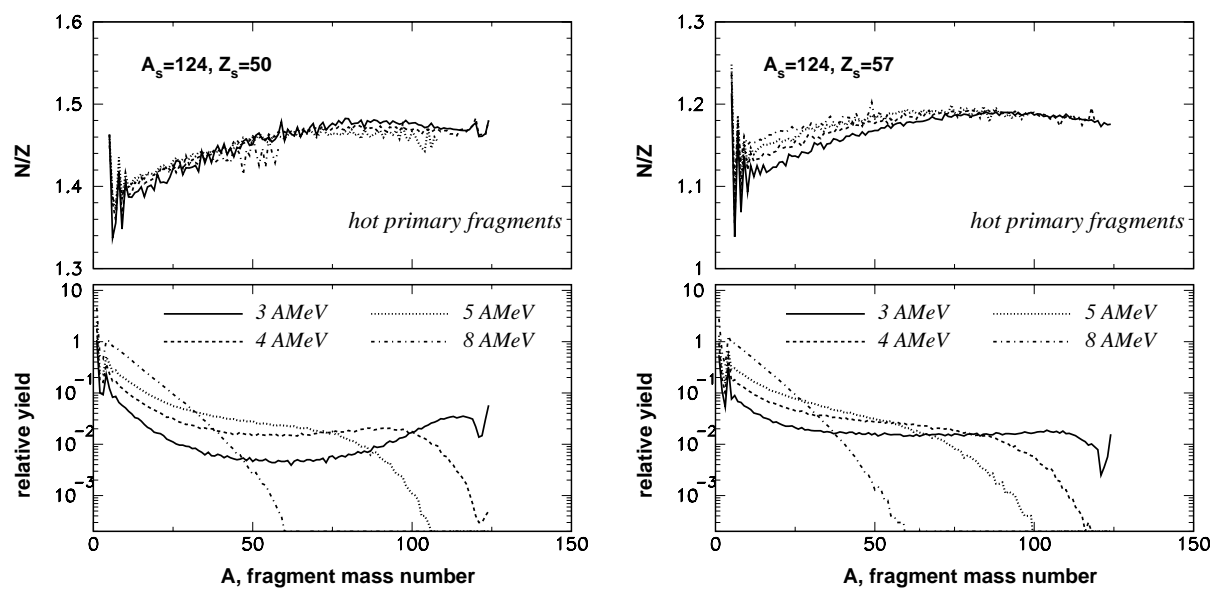

Figure 1. Neutron-to-proton ratios $N / Z$ (top panels) and mass-yield curves (bottom panels) of the primary hot fragments produced at the breakup of two systems with fixed mass number $A=124$ according to SMM calculations. The lines correspond to four excitation energies between 3 and $8 \mathrm{MeV}$ per nucleon as indicated. Note the difference of the ordinate scales in the top panels (from Ref. [10]).

multifragment decays.

In a series of experiments [11,12], multifragment decay of projectile spectators has been studied with the ALADiN forward-spectrometer at the SIS accelerator (GSI). The primary projectile spectator emerging from the abrasion phase is a well defined source which can be reconstructed on an event-by-event basis from the multiplicity and energy of the fragments. The isotropy of the fragment emission in the decaying spectator rest-frame suggests an emission from a thermodynamical equilibrated source. In these collisions, energy depositions are reached, which cover the range from particle evaporation to multifragment emission and further to the total disassembly of the system, the so-called Rise and Fall of multifragment emission [11] (Fig. 2). The most prominent feature of the multi-fragment decay is the universality of the fragment multiplicites and the fragment charge correlations. These observables are invariant with respect to the entrance channel, i.e. independent of the beam energy and the target or projectile masses, if plotted as a function of $Z_{\text {bound }}$. This latter quantity, being the sum of the atomic numbers $Z_{\mathrm{i}}$ of all projectile fragments with $Z_{\mathrm{i}}>1$, represents a measure of the size of the spectator and, in a geometrical picture, is monotonously correlated with the impact parameter. The loss of memory of the entrance channel is an indication that equilibrium is attained prior to the fragmentation stage of the reaction. It will be interesting to investigate whether the observed universality of spectator decays includes the invariance with isospin.

Besides the isospin, also the mass of the system may play an important role for fragmentation observables of the reaction. It has been suggested that the dependence of the breakup temperature on the excitation energy (caloric curve) is governed by the limiting 


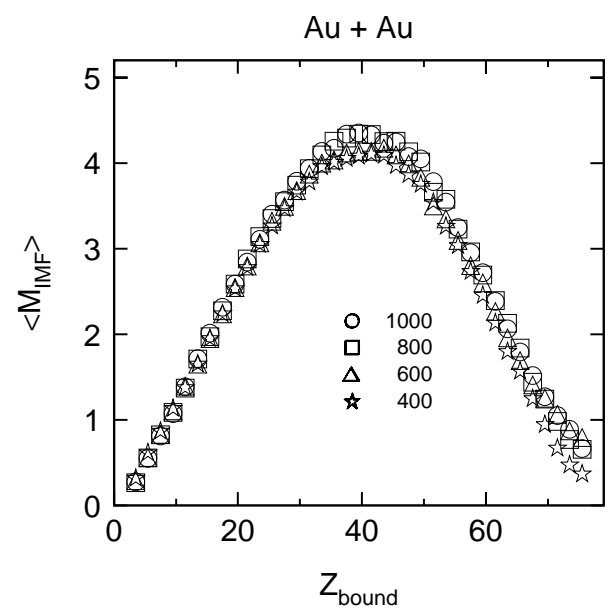

Figure 2. Rise and Fall of multifragment emission [11]: the mean multiplicity of intermediate mass fragment is plotted as a function of $Z_{\mathrm{bound}}$ for different energies.

temperature $[13,14]$. This quantity represents the maximum temperature at which nuclei are found to exist as self-bound objects in Hartree-Fock calculations [15]. In lighter systems the limiting temperature is higher, mainly so because the Coulomb energy is reduced (Fig. 3). On the other hand, SMM calculations predict nearly mass-invariant temperatures for the coexistence region [9]. The comparison of two systems with different mass should, therefore, permit distinguishing whether the breakup temperature is determined by the binding properties of the excited hot nuclear system or by the phase space accessible to it by fragmentation. The same test can be made by varying the isospin.

\section{The Experimental Setup}

The most recent ALADiN experiment has been devoted to investigating isotopic effects in the decay of projectile spectators at relativistic energies. In order to extend the range of isotopic compositions of the excited spectator systems, secondary beams have also been used. This and the clean separation of the spectator sources in rapidity make this type of reaction unique for studying the isospin dependence of nuclear multifragmentation.

Four different projectiles, all with an incident energy of $600 \mathrm{AMeV}$, have been investigated allowing a study of various combinations of masses and $N / Z$ ratios in the entrance channel: ${ }^{124} \mathrm{Sn},{ }^{197} \mathrm{Au},{ }^{124} \mathrm{La}$ and ${ }^{107} \mathrm{Sn}$. The two latter beams have been delivered by the FRagment Separator (FRS) of the GSI as products of the fragmentation of a primary ${ }^{142} \mathrm{Nd}$ beam at $1.1 \mathrm{AGeV}$ on a ${ }^{9} \mathrm{Be}$ production target. The necessity of low beam intensities for the best operational condition of the ALADiN setup $(\approx 2000$ particles/sec), and the possibility of using a thick target in order to achieve high interaction rates are indeed conditions compatible with radioactive-ion-beam experiments. Moreover, the in- 


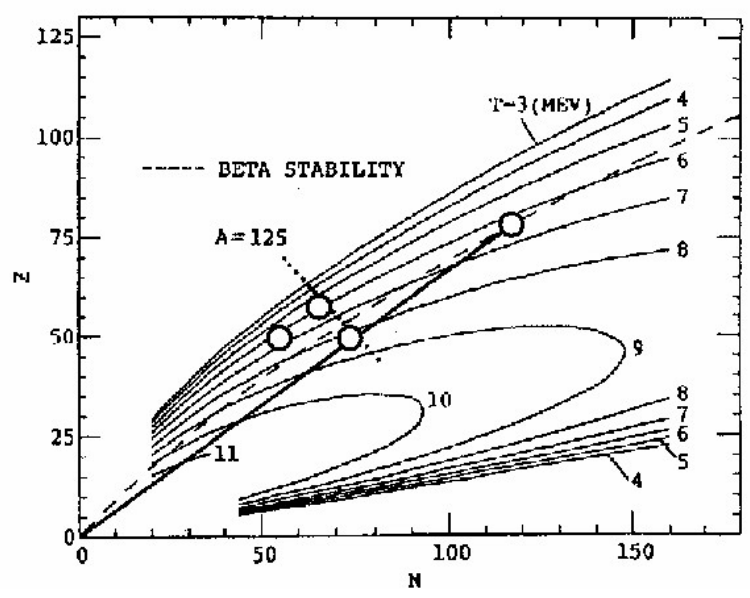

Figure 3. Location of the four studied projectiles in the plane of atomic number $Z$ versus neutron number $N$. The contour lines represent the limiting temperatures according to Ref. [15], the dashed line gives the valley of stability, and the full line corresponds to the $N / Z=1.49$ of ${ }^{197} \mathrm{Au}$.

verse kinematics offers the possibility of a threshold-free detection of all heavy fragments and residues and thus gives a unique access to the breakup dynamics.

A cross sectional view of the used setup is shown in Fig. 4. The beam enters from the left and passes thin time- and position-detectors before reaching the ${ }^{\text {nat }} \mathrm{Sn}$ target with $500-\mathrm{mg} / \mathrm{cm}^{2}$ areal density. The isotopic composition of the secondary beams was determined and monitored from the magnetic rigidity measured at the FRS, from a velocity measurement along the 80-m flight path between the FRS and the ALADiN setup, and from the charge measurement with the TP-MUSIC IV detector.

Projectile fragments entering into the acceptance of the magnet are tracked and identified in the TP-MUSIC IV detector and in the time-of-flight (TOF) wall. Neutrons emitted in directions close to $\theta_{\text {lab }}=0^{\circ}$, are detected with the Large-Area Neutron Detector (LAND). The dash-dotted lines represent the beam directions before and after the deflection by $7^{\circ}$ in the field of the ALADiN magnet.

The measurement of the charge and the momentum vector of all projectile fragments with $Z \geq 2$ has been performed with high efficiency and high resolution with the TPMUSIC IV detector. In order to cover the wide dynamic range necessary to measure nuclei from $\mathrm{He}$ up to $\mathrm{Au}$ with the best possible resolution, two different kinds of detectors are connected to the field cage of the TP-MUSIC detector on either side [16]: the ionization charge collected at 24 anodes provides optimum $Z$ resolution for heavy fragments $(Z>8)$, whereas the 3D tracking information of all particles and the charges of lighter fragments are obtained from 4 position-sensitive proportional counters. The position of the ionizing particles in the non-bending plane is determined from the position along the proportional 


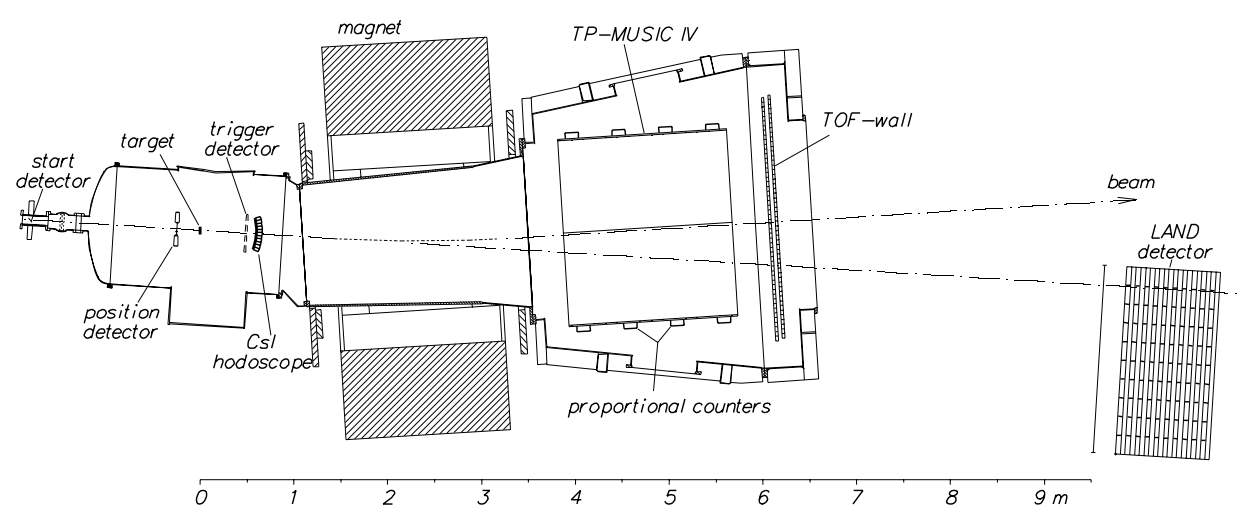

Figure 4. Cross sectional view of the ALADIN setup.

counters, whereas the position in the bending plane is determined by measuring the total drift time of the electrons to the detectors. In order to improve the performance of the TPMUSIC detector, an upgrade has been undertaken [17] which involved the construction of a new set of proportional counters and a redesign of all components of the electronic chain compared with the previous version of the detector [16]. The proportional counters use a combination of charge-division and pad-readout techniques to reconstruct the position in the non-bending plane of the tracks of nuclei [17]. The pads of each section are connected modulo five: the resulting position ambiguity can indeed be resolved by using the less precise position information obtained from the anode wires with the charge-division method.

To extract the signals from each sector of the proportional counters, seven charge-sensitive preamplifiers are used. The signals of the preamplifiers, after removal of the highfrequency component through an anti-aliasing filter, are digitized by 14-bit Flash ADC's without prior shaping. The typical noise is of 1 Least Significant Bit (standard deviation) over a dynamic range of $1: 10^{4}$. The output, generated at a rate of up to $40 \mathrm{MHz}$, is stored and processed by a system containing FPGA and DSP chips.

Using the reconstructed values for the rigidity and path length, the charge of the particle measured by the TP-MUSIC detector, and the time of flight given by the TOF-wall, the velocity and the momentum vector can be calculated for each detected charged particle. The knowledge of velocity and momentum allows then the calculation of the particle's mass. Mass spectra for the ${ }^{124} \mathrm{Sn}$ and ${ }^{124} \mathrm{La}$ fragmentation are shown in Fig. 5. Single mass resolution for charges up to 12 is obtained, corresponding to a mass resolution $\triangle A / A$ of approximately $4.0 \%$ (FWHM) for light fragments.

Already by inspection of the mass spectra, first order isotopic effects can be observed: in the case of the neutron-rich system higher yields are obtained for the neutron-rich isotopes. 

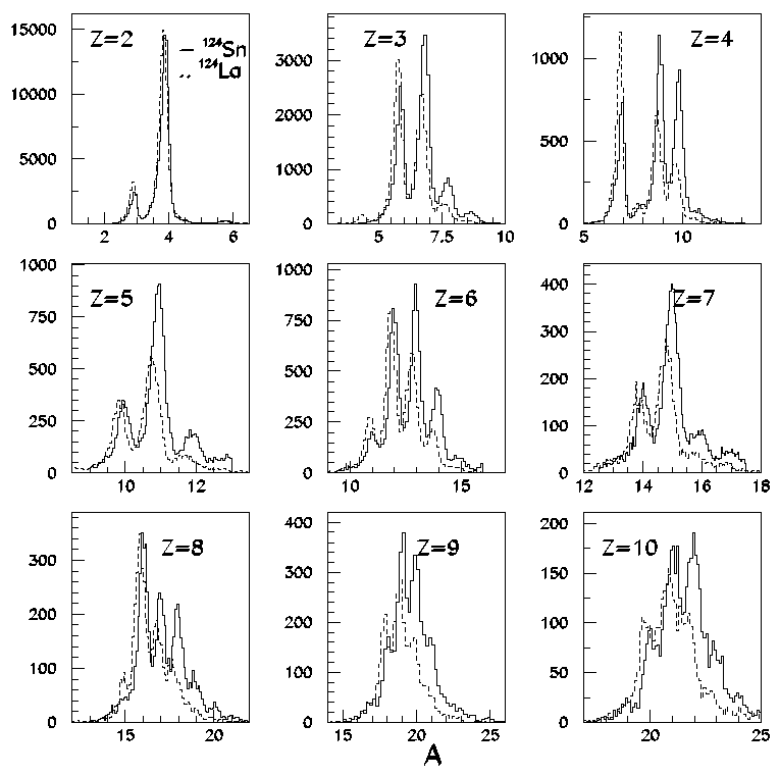

Figure 5. Mass spectra for light fragments with $Z \leq 10$ from the fragmentation of ${ }^{124}$ La (dashed line) and ${ }^{124} \mathrm{Sn}$ (full line).

\section{Gross Properties of the multifragment decay}

In order to investigate to which extent the isotopic composition of the excited spectator affects the gross properties of the multifragmentation pattern, charge partitions and multiplicity distributions have been analyzed, as well as the mean $N / Z$ of medium size fragments, and the results have been compared with the SMM prediction.

In Fig. 6, the obtained correlation between the mean multiplicity of intermediate-mass fragments, $\left\langle M_{I M F}\right\rangle$, and the variable $Z_{\text {bound }}$ for the ${ }^{107} \mathrm{Sn},{ }^{124} \mathrm{Sn}$ and ${ }^{124} \mathrm{La}$ systems is shown (left panel). The global universality of the Rise and Fall behavior is preserved, but already some distinct differences can be observed. At small excitation energies (large $Z_{\text {bound }}$ values) the curves end, as expected, approximately at the charge of the original projectiles. However, the slope of the curve is steeper in the case of the ${ }^{124} \mathrm{Sn}$. This effect can be understood by considering that in the case of the neutron-rich system, heavy residues with low excitation energy will predominantly emit neutrons, a channel that is suppressed in the case of the two neutron-poor nuclei. In these latter cases, peripheral collisions are more spread out towards smaller values of $Z_{\text {bound }}$, thus leading to a slower rise of $\left\langle M_{I M F}\right\rangle$. This effect, as well as the corresponding difference in the $Z_{\text {bound }}$ distribution, is in good agreement with the SMM predictions.

Going towards more central collisions, we observe a lower maximum in $\left\langle M_{I M F}\right\rangle$ for the lighter $\mathrm{Sn}$ isotope, whereas the two $\mathrm{A}=124$ systems exhibit the same value for the mean 

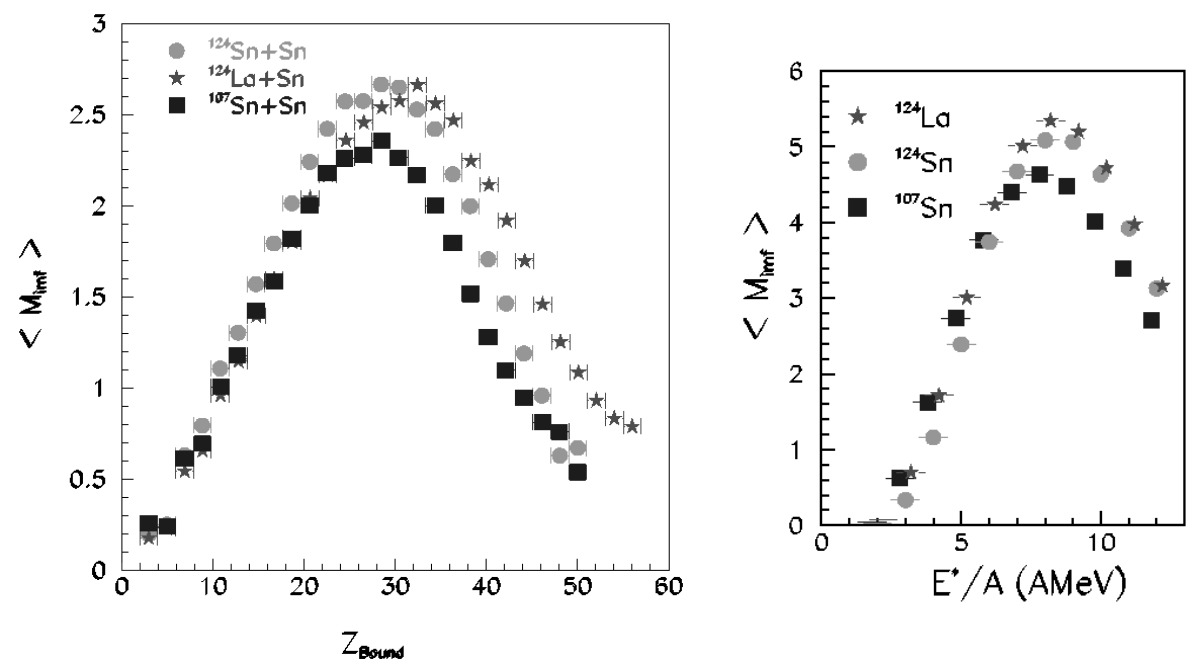

Figure 6. Left Panel: Experimental Rise and Fall of multifragmentation correlating the mean multiplicity of intermediate-mass fragments and $Z_{\mathrm{bound}}$. Right Panel: Prediction of the Statistical Multifragmentation Model for the mean IMF multiplicity as a function of the excitation energy for excited ${ }^{124} \mathrm{La},{ }^{124} \mathrm{Sn}$ and ${ }^{107} \mathrm{Sn}$ nuclei.

multiplicities. This mass effect, reproduced by SMM calculations (Fig. 6 right panel) is rather intriguing since the definition of $\operatorname{IMF}(3 \leq Z \leq 20)$ is based on the charge. Therefore, the partition space should be primarily a function of the charge of the source. In the rise, up to 6 or $7 \mathrm{MeV}$ per nucleon, the number of fragments is just given by the excitation energy. The ${ }^{107} \mathrm{Sn}$ bends over more quickly towards vaporization: less neutrons are available and we may have more alpha-type fragments (like ${ }^{8} \mathrm{Be},{ }^{12} \mathrm{C}$ ) that decay easily into alpha particles. The absolute multiplicities are overpredicted because the calculations are performed for sources of fixed mass and not for the ensemble of spectator systems produced in the collision. Very specific isotopic effects, even though small, can also be extracted from the analysis of the mean $N / Z_{\mathrm{s}}$ for medium-size fragments. In this case, in order to generate two data samples selected by excitation energy, two cuts on the maximum charge detected in each event $\left(Z_{\max }\right)$ have been used: the data have been correspondingly sorted into two different bins of the variable $Z_{\max }$, corresponding to high and low excitation energies. The mean $N / Z$ s for medium-size fragments obtained are shown in Fig. 7. For the ${ }^{124} \mathrm{Sn}$, the values are always bigger than the ones obtained in the case of the ${ }^{124} \mathrm{La}$ : this observation reflects simply the difference in the $N / Z$ of the original projectiles. In the case of the ${ }^{124} \mathrm{La}$, on the other hand, a difference in the mean $N / Z$ s corresponding to the two cuts is observed, whereas the ${ }^{124} \mathrm{Sn}$ shows almost no sensitivity. Also this characteristics is predicted by the SMM calculations as evident from Fig. 1. This latter case, however, refers to hot primary fragments whereas the extracted $<N / Z>$ correspond to the detected final fragments: this explains why the absolute scale 

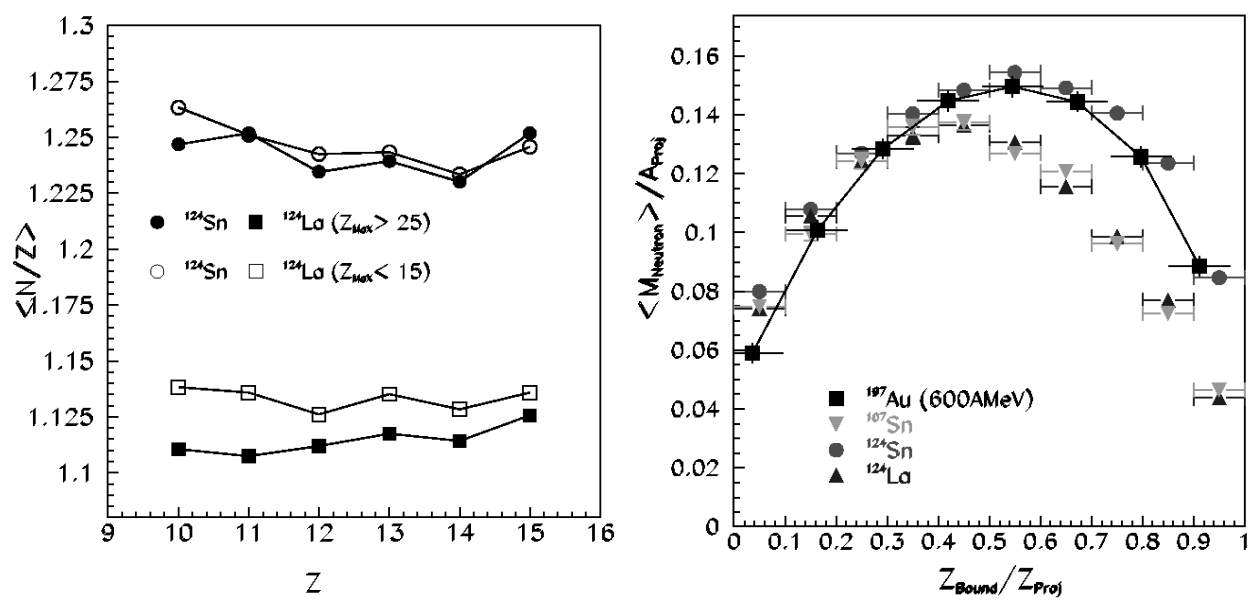

Figure 7. Left Panel: Mean $N / Z$ distribution for medium-size fragments for two cuts in the variable $Z_{\max }$ (corresponding to two different excitation energy intervals). Right Panel: Preliminary mean neutron multiplicity, as derived from the raw hit multiplicity in Land, corrected for solid angles and scaled for projectile mass as a function of $Z_{\mathrm{bound}}$ for the three analyzed systems compared with the data for ${ }^{197}$ Au projectile fragmentation [18].

in the data is much lower than the predicted one. It is, however, interesting to see that this feature seems to survive the sequential decays.

In the statistical scenario, this difference arises from the dependence of the number of neutrons which will have to be carried by the light fragments on the yields of heavy fragments and their capability of carrying neutrons (depends on mass and $N / Z$ ). This effect supports the idea of overall equilibrium of the system at breakup.

\section{Neutron Multiplicities}

As already mentioned, the used experimental setup allowed also neutron detection. In Fig. 7 the mean neutron multiplicity, corrected for solid angle and scaled for projectile mass, has been correlated with the variable $Z_{\text {bound }}$. A very clear isotopic effect (again first-order effect) is visible: more neutrons are produced in the case of the neutron-rich system. In particular a good agreement has been found by comparing the scaled neutron multiplicity measured for the ${ }^{124} \mathrm{Sn}$ system with the one obtained in the case of the heavier ${ }^{197} \mathrm{Au}$ projectile [18], which has the same $N / Z$. This difference decreases with excitation energy and the curves nearly merge. Neutrons will be important for establishing the mass and energy balance, in particular for calorimetry. In this respect, it is crucial to identify the spectator neutrons and to distinguish them from the fireball ones.

Moreover in the grand-canonical approximation it can be demonstrated that, from the ratio of the neutron yields the symmetry term of the nuclear equation of state can be determined once the temperature and the isotopic composition of the systems are known [19]. 
In this respect, neutron analysis could allow to investigate the symmetry-term dependence on the excitation energy of the system, in a similar way as with the isoscaling analysis [20].

\section{Conclusions}

First preliminary results from the most recent ALADiN experiment devoted to the investigation of mass and isospin effects in multifragmentation have been reported. Global quantities as, e.g., the mean multiplicity of intermediate-mass fragments exhibit small but significant variations with the isotopic composition of the fragmenting projectile.

Much larger effects are observed for the $N / Z$ ratios of the emitted fragments. Their characteristic dependence on excitation energy promises to allow a meaningful test of the attainment of statistical equilibrium.

The measured neutron yields have a maximum at intermediate values of $Z_{\text {bound }}$, practically coinciding with the maximum fragment multiplicity. Their isotopic dependence is large for large $Z_{\text {bound }}$ but gradually disappears with decreasing $Z_{\text {bound }}$, i.e. for the highest excitation energies.

The authors would like to thank the staff of the GSI for providing heavy ion beams of the highest quality and for technical support. C.Sf. acknowledges the receipt of an Alexandervon-Humboldt fellowship. This work was supported by the European Community under contract No. HPRI-CT-1999-00001 and by the Polish Scientific Research Committee under contract No. 2P03B11023.

\section{REFERENCES}

1. H. Müller and B.D. Serot, Phys. Rev. C 52 (1995) 2072.

2. I. Bombaci and U. Lombardo, Phys. Rev. C 44 (1991) 1892.

3. A.B. Larionov et al., Nucl. Phys. A 658 (1999) 375.

4. R. Wada et al., Phys. Rev. Lett. 58 (1987) 1829.

5. H.W. Barz et al., Phys. Lett. B 211 (1988) 10.

6. D. Hahn and H. Stöcker, Nucl. Phys. A 476 (1988) 718.

7. H.S. Xu et al., Phys. Rev. Lett. 85 (2000) 716.

8. P.M. Milazzo et al.,Phys. Rev. C 62 (2000) 041602(R).

9. J.P. Bondorf et al., Phys. Rep. 257 (1995) 133.

10. A.S. Botvina and I.M. Mishustin, Phys. Rev. C 63 (2001) 061601.

11. A. Schüttauf et al., Nucl. Phys. A 607 (1996) 457.

12. J. Pochodzalla et al., Phys. Rev. Lett. 75 (1995) 1040.

13. J.B. Natowitz et al., Phys. Rev. C 52 (1995) R2322.

14. J.B. Natowitz et al., Phys. Rev C 65 (2001) 034618.

15. J. Besprosvany and S. Levit, Phys. Lett. B 217 (1989) 1.

16. G. Bauer et al. Nucl. Inst. and Meth. A 386 (1997) 249.

17. C. Sfienti et al., in Proceedings of the $X L I^{\text {st }}$ International Winter Meeting on Nuclear Physics, Bormio, Italy, 2003, ed. by I. Iori, A. Moroni, Ricerca Scientifica ed Educazione Permanente Suppl. \# 120 (Milano 2003), p. 323.

18. E. Zude, Ph.D. Thesis, Universität Frankfurt, 1995.

19. A.S. Botvina et al., Phys. Rev. C 65 (2002) 044610.

20. A. Le Févre et al., nucl-ex/0409026. 